\title{
Role of fiberoptic bronchoscopy in the diagnosis of pulmonary infiltrates in patients with hematological malignancies
}

\author{
Yosri Mohamed Kamel Akl ${ }^{\mathrm{a}}$, Hamdi Mohamed El Zawam ${ }^{\mathrm{b}}$, \\ Reem Ibrahim Mohamed ElKorashy ${ }^{a}$, Mohamed Said Ismail ${ }^{a}$, \\ Amir Kamal Morris Hanna ${ }^{a}$
}

\begin{abstract}
Rationale Patients with hematological malignancies are a special challenge to health care providers as they undergo a wide variety of immune-suppressive therapies. Both disease and therapy can cause complications. The lungs may be directly injured through infectious or toxic insults. Early identification of the nature of infiltrates will result in better outcome in the management of these patients. Fiberoptic bronchoscopy (FOB) may be a good tool for indentifying the infiltrate nature by using different diagnostic techniques like transbronchial lung biopsy (TBLB) and bronchoalveolar lavage (BAL).
\end{abstract}

Patients and methods This study was performed over 6 months, in Kasr Al-Aini Hospital, Cairo University. It included 30 patients with hematological malignancies presenting with pulmonary infiltrates confirmed by computed tomography of the chest. All patients underwent history taking, clinical examination, and investigations included complete blood count and coagulation profile, BAL for culture and sensitivity, cytology examination, and TBLB.

Results Overall diagnostic yield of FOB was $73.3 \%$ in this study. The diagnostic yield of FOB was higher for infectious $(43.3 \%)$ than both noninfectious $(20 \%)$ and mixed $(10 \%)$ etiologies. The combined diagnostic yield of BAL for bacteriological examination and TBLB was higher than that of

\section{Introduction}

Hematological malignancies are cancers of the hematopoietic cell lines. They include acute and chronic leukemias, Hodgkin's disease, nonHodgkin's lymphomas (NHLs), multiple myeloma, and myelodysplastic syndrome [1].

Immunosuppressive drugs may have impressive response in terms of disease-free survival [2], yet complications can be substantial [3]. Moreover, pulmonary complications related to the disease process differ for different cancers and therapies [4].

Multidisciplinary approach is required to manage these pulmonary complications [5], which may be of short term, necessitating intensive care treatment [6]. Diagnosis of pulmonary infiltrates can be achieved by chest radiography, serological testing, sputum analysis, and bronchoscopic techniques, including bronchoalveolar lavage (BAL) [7].

Despite being invasive, the diagnostic role of the fiberoptic bronchoscopy (FOB) remains controversial [8]. Some additional procedures such as transbronchial
BAL for cytology examination. Bacteriological examination revealed a predominance for gram-negative bacteria in $62.5 \%$ of total culture and sensitivity.

Conclusion This study supports the role of FOB as the initial procedure of choice for identifying the nature of pulmonary infiltrates in patients with hematological malignancies. Because our results suggest that BAL and TBLB are complementary, we recommend performing TBLB whenever it is deemed safe. On the contrary, cytological examination of BAL fluids was not helpful in reaching a final diagnosis.

Egypt J Bronchol 2018 12:340-345

(C) 2018 Egyptian Journal of Bronchology

Egyptian Journal of Bronchology 2018 12:340-345

Keywords: fiberoptic bronchoscopy, hematological malignancies, pulmonary infiltrates

aPulmonology Department, ${ }^{b}$ Oncology Department, Cairo University, Egypt

Correspondence to Mohamed Said Ismail, MD Pulmonary Medicine,

Department of Pulmonary Medicine, Cairo University Hospitals, Kasralainy Street, 11562, Egypt. Tel.: 00201110014998 ;

e-mail: mohammed.hassan@kasralainy.edu.eg

Received 18 October 2017 Accepted 16 January 2018

lung biopsy (TBLB) can supply tissue samples, which may allow the identification of the nature of the process [9].

\section{Rationale}

The aim of this study was to detect the role of FOB procedures in diagnosis of pulmonary infiltrates in patients with hematological malignancies.

\section{Patients and methods}

A prospective cohort study was performed in Chest Department in collaboration with Oncology Department, Kasr Al-Aini Hospital, Cairo University over 6 months. The study included all patients with hematological malignancies who presented with pulmonary infiltrates, who were above the age of 18 years, who were of either sex, and who signed a written informed consent. Exclusion criteria included

This is an open access journal, and articles are distributed under the terms of the Creative Commons Attribution-NonCommercial-ShareAlike 4.0 License, which allows others to remix, tweak, and build upon the work non-commercially, as long as appropriate credit is given and the new creations are licensed under the identical terms. 
all contraindications of performing FOB like profound refractory hypoxemia, severe bleeding diathesis contraindicating bronchoscope and/or biopsy, malignant cardiac arrhythmias, lack of patient cooperation, recent myocardial infarction or unstable angina, respiratory insufficiency or failure, uremia, or unstable asthma. Full medical history, clinical examination, and routine investigations were performed.

High-resolution computerized tomography (HRCT) of the chest was done to confirm the presence of pulmonary infiltrates. FOB (Pentax FB-1V, Asahi Optical Co., Tokyo, Japan) was done, and BAL was performed for examination of aerobic and anaerobic culture and sensitivity, fungal culture, direct smear stained by Ziehl-Neelsen stain for acid-fast bacilli, and silver stain if Pneumocystis jiroveci pneumonia is suspected. BAL (average sample of $\sim 50 \mathrm{ml}$ ) for cytological examination and TBLB were performed using the cupped forceps, with a size $3 \mathrm{~mm}$ for the biopsy, with average $3-5$ biopsies [10].

\section{Statistical analysis}

Data were analyzed by the statistical package for the social science software, version 20 (IBM Corp, Armonk, New York, USA). Descriptive results were expressed as mean, and non-numerical data were expressed as number and percentage. Graphics were performed using Microsoft excel 2010. $\chi^{2}$-Study of the relationship between variables was performed using Pearson's correlation.

\section{Results}

The study population included 30 patients with hematological malignancies and pulmonary infiltrates in the HRCT, who were older than 18 years. Their primary disease diagnoses are presented in Table 1. Leukemia accounted for $83.3 \%$ of cases, with male : female ratio of $3: 2$, whereas the rest of the cases (16.7\%) were diagnosed as having lymphoma, with

Table 1 Primary disease illustration

\begin{tabular}{lc}
\hline Diagnosis of infiltrates & $n(\%)$ \\
\hline Leukemia & \\
ALL & $5(16.7)$ \\
AML & $12(40.0)$ \\
CLL & $4(13.3)$ \\
CML & $4(13.3)$ \\
Lymphoma & \\
HL & $2(6.7)$ \\
NHL & $3(10)$ \\
Group total & $30(100.0)$ \\
\hline
\end{tabular}

ALL, acute lymphocytic leukemia; AML, acute myeloid leukemia; CLL, chronic lymphocytic leukemia; CML, chronic myeloid leukemia; HL, Hodgkin's lymphoma; NHL, non-Hodgkin's lymphoma. male : female ratio of $2: 3$. The total study population showed male percentage of $56 \%$, whereas the female percentage was $44 \%$.

The mean age for the study population was $46 \pm 10.1$ years. Mean age for lymphoma cases was $45.8 \pm 12.2$ years, whereas for leukemia cases was $46 \pm 9.9$ years.

The HRCT showed different radiological features (Fig. 1), usually presented in combinations in the same patient.

Significant positive correlation was seen between the consolidation pattern in the chest computed tomography (CT) and infection etiology, with $P$ value of 0.05 .

Moreover, positive correlation was seen between the mass lesion in the chest $\mathrm{CT}$ and malignant etiology, with $P$ value 0.04 . The rest showed nonsignificant correlations with etiology.

The patients were then subjected to FOB, where BAL and TBLB were obtained. BAL was examined for culture and sensitivity for aerobic and anaerobic bacteria and fungal infection. Moreover, BAL was sent for direct smear staining by Zeihl-Neelsen stain for acid-fast bacilli, which was negative in all cases.

Cultures showed positive results in $50 \%$ of the cases $(n=15)$. Results showed gram-positive infection in $13.3 \%(n=2)$ of the cases, gram-negative infection in $66.7 \%(n=10)$, and fungal infection in $20 \%(n=3)$, including a case of $P$. jiroveci.

However, in the final etiological diagnosis (Table 2), the total number of cases of infections were considered 16 , with a total percentage of $53.33 \%$ of the total study population. This case showed negative BAL culture, acellular smear in BAL cytology, and radiological finding suggestive of infection (consolidation with air bronchogram and tree in bud sign). The patients

Figure 1

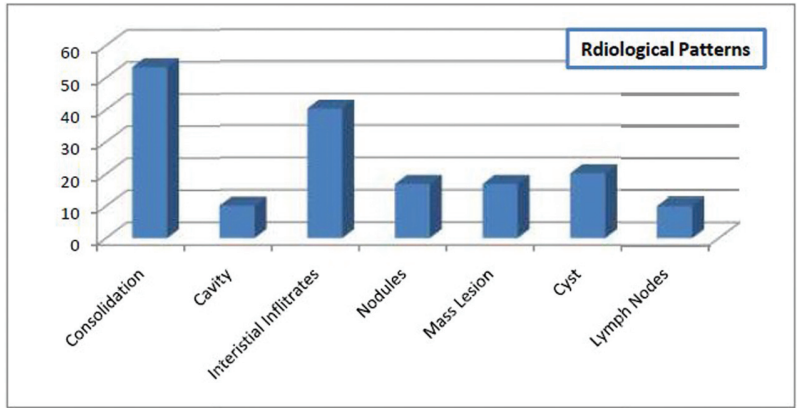

Different radiological pictures in chest computed tomography. 
Table 2 Final etiological diagnosis

\begin{tabular}{lccccc}
\hline & \multicolumn{2}{c}{ Etiology $[n(\%)]$} & \multicolumn{1}{c}{ Group total $[n(\%)]$} \\
\cline { 2 - 4 } & Infectious & Noninfectious & Mixed & Undiagnosed & $5(16.7)$ \\
Lymphomas & $0(0)$ & $2(33.3)$ & $1(33.3)$ & $2(25.0)$ & $25(83.3)$ \\
Leukemias & $13(100.0)$ & $4(66.7)$ & $2(66.7)$ & $6(75.0)$ & $30(100.0)$ \\
Total & $13(100.0)$ & $6(100.0)$ & $3(100.0)$ & $8(100.0)$ & 3 \\
\hline
\end{tabular}

finally showed clinical and radiological improvement on empirical broad-spectrum antibiotics.

The predominant gram-negative bacteria present was further analyzed to show Klebsiella spp. infection in $40 \%(n=4)$ of the total gram-negative infected cases, followed by Enterobacter spp. infection 20\% $(n=2)$ and others.

Cytological examination of the BAL revealed (Fig. 2) that most of the specimens (56.7\%) were inflammatory in nature without predominant cell type $(n=17)$. Those with predominant lymphocytic cell type represented $23.3 \% \quad(n=7)$, and smears associated with atypical or malignant cells were $16.7 \%(n=5)$. Only one acellular smear was seen (3.3\%), which is considered one of the pitfalls of cytology regarding the way of storage.

During the procedure, TBLB was performed for 22 patients only. The rest of the patients did undergo TBLB owing to complications in the form of severe hemoptysis (five patients) or dangerous hypoxia (three patients), which necessitated termination of the procedure.

TBLB showed different pictures of pathological changes (Fig. 3) starting from acute lung injury associated with Pneumocystis pneumonia $(n=1)$, to inflammatory cellular infiltrate and alveolar consolidation associated with infection $(n=12)$. Picture of malignancy owing to progression of the disease was illustrated as myeloid infiltration, leukemic infiltration, hyperchromatic atypical cells, or lymphomas of any type $(n=7)$. Complication of chemotherapy was presented by interstitial pneumonitis and toxic pneumonitis with organizing pneumonia $(n=2)$.

After performing the procedures - mentioned before - through the FOB and evaluating the results, it was easier to categorize the different etiologies for the pulmonary infiltrates that occur in patients with hematological malignancies, as shown in Fig. 2.

Thus, the overall diagnostic yield of FOB was deduced to be $73.3 \%$ in this study.

\section{Figure 2}

Percentage of different pathological patterns in TBLB

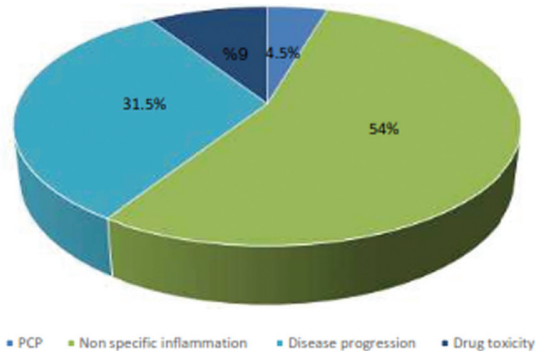

Bronchoalveolar lavage cytology results. BAL, bronchoalveolar lavage.

Figure 3

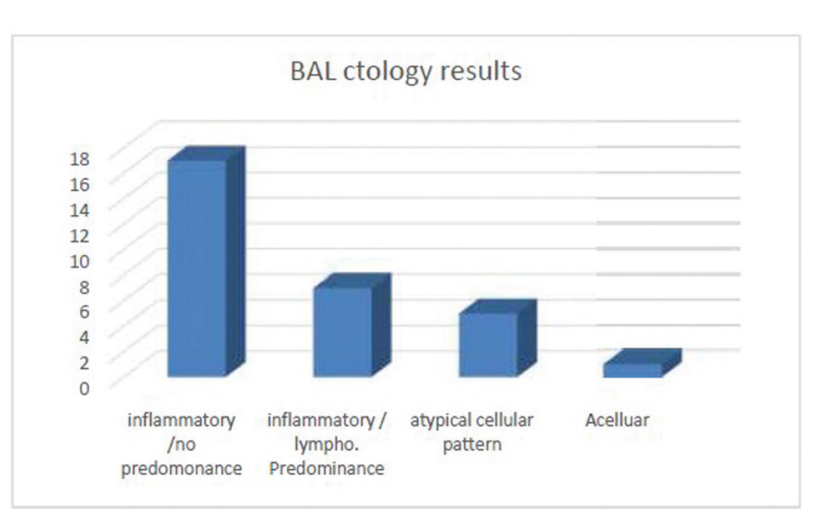

Percentage of different pathological patterns in transbronchial lung biopsy. PCP, pneumocystis pneumonia.

\section{Discussion}

This study confirms the important diagnostic role of FOB in immunocompromised patients with lung infiltrates.

According to Table 1, most patients were diagnosed as having leukemia (83.3\%), whereas patients with lymphoma represented $16.7 \%$. This was different from the incidence mentioned in American Cancer Society, 2014 [11] where lymphoma was found in 63.1\%, whereas leukemia was found in 36.9\%. However, a single center Egyptian National Cancer Registry for the year 2008 stated that the number of leukemias : lymphoma was $12: 5$. It also considered leukemia as one of the frequent cancers, whereas lymphomas, both Hodgkin's and non-Hodgkin's, 
were considered infrequent [12]. On comparing the incidence rates of lymphoma and leukemia in three different regions in Egypt, (upper, middle, and lower Egypt), there were no specific geographical pattern; even the incidence rates were different from one region to the other [13]. This may reflect regional or ethnic differences $[13,14]$. This incident rate may also be a reflection of increased incidence of respiratory illness in patients with leukemia [15], which necessitated the patients to seek pulmonologists, thus they were enrolled in the study.

It was found that the mean age of patients with lymphoma was $45.8 \pm 12.2$ years, whereas the median age stated by Smith et al. [16] was 67.2 (54.9-76.5) years. In patients with leukemia, the mean age was 46 \pm 9.9 years. It was found that for each type of leukemia, age group distribution is completely different from one to the other group, as median age in CLL is $65-70$ years [17], whereas ALL is considered to have bimodal age distribution, where the higher incidence is in children 2-5 years and then another less incident peak is at age older than 50 years [18].

The current study pointed out that the etiology of pulmonary infiltrates can be either infectious, noninfectious, or mixed (Table 2). The diagnosis was reached in $22(73.3 \%)$ patients, whereas eight (26.7\%) patients remained undiagnosed. Noninfectious cases were either recent disease progression or chemotherapy toxicity.

The diagnostic yield of FOB in immunocompromised patients with lung infiltrates varied from 15 to $93 \%$ in prior studies [19]. In this context, the diagnostic yield of FOB in the current study was $73.3 \%$, which is similar to that reported by some investigators. Heterogeneity in patient populations in different studies appears to be the most likely explanation for the wide variation in the reported yield of FOB. For example, because FOB has a higher diagnostic yield for infectious causes of lung infiltrates, studies that enrolled a greater proportion of patients with lung infections reported a higher diagnostic yield than studies with a higher proportion of patients with noninfectious causes of lung infiltrates [19]. This was obvious in the current study which showed higher diagnostic yield for infections only (43.3\%) than for the noninfectious causes (20\%) and mixed causes (10\%).

In a trial to correlate the commonest radiological finding with the etiological diagnosis as shown in (Fig. 2), it was found that most patients with infectious etiology (ten of 13 patients) had consolidation in the $\mathrm{CT}$ chest. This finding was significant $(P=0.05)$, whereas ground glass and mosaic infiltrates and mass lesions were encountered in the noninfectious etiology. Although imaging study findings cannot prove the nature of the pulmonary disease, yet it may provide valuable clues as consolidation was found to be the most encountered feature in bacterial pneumonia, whereas ground glass opacities and mosaic pattern were more common in drug-induced toxicity and disease progression [20].

The current study showed that culture was positive in $50 \%$ of the studied cases. Predominance of gramnegative bacteria (66.7\%) was observed. The main organism identified as a cause of bacterial pneumonia was Klebseilla spp. It was stated that in neutropenic patients, gram-negative bacteria are usually the predominant cause of infection in isolated bronchoscopic specimens [21], usually pseudomonas was the most commonly isolated bacteria, whereas in acute lung injury, there was found increased incidence of gram-positive bacteria, such as Staphylococcus aureus, Enterococcus faecium, and group A Beta hemolytic streptococcus [22]. However, according to Kotloff et al. [23], gram-negative organisms may be the etiology in up to $25 \%$ of patients.

Our speculation for the increased incidence of the gram-negative bacteria relative to the gram-positive bacterial infection was related to the alteration of the oropharyngeal microbiome, leading to lower respiratory tract infections. This could be related to the recurrent hospital admission, lowered immunity, stress, malnutrition, and debilitation [24].

Incidence of fungal infection was found to be $20 \%$ of all infections, and $10 \%$ of the study population. This was close to the findings from another study where fungal infection was diagnosed in nine of 51 cases (18\%) [25].

According to the current study, cytology was not helpful in the diagnosis of infiltrates, because more than $50 \%$ of the cases showed inflammatory smear with no specific cell predominance, and even the cases with predominant lymphocytes showed no relation with the infiltrate etiology. Studies showed controversial results regarding the presence of lymphocytic predominance in BAL, which ranges from $100 \%$ in some [26] to a range of 30-36\% in others [27]. Even the detection of atypical cells was not helpful, which was in agreement with some authors [28] and contradictory to others, where one found that $67 \%$ of 15 cases of NHL and three of nine cases of Hodgkin's lymphoma were 
identified by the presence of characteristic atypical cells in the cytology [29], and the other observed atypical cells in $45 \%$ of patients with pulmonary B-NHL [26]. It seems that the different forms of hematological malignancies have different ranges of diagnosis with BAL cytology, where the diagnostic yield of BAL for the diagnosis of Hodgkin's lymphoma seems to be below 33\% [30]. Blast cells in BAL fluid could be difficult to interpret when the peripheral blood blast cell count is high, as BAL fluid might be contaminated by peripheral blood cells [31]. Reports of the contribution of BAL to the diagnosis of pulmonary leukemic infiltration can therefore be considered anecdotal [32].

On the contrary, one case had acellular smear. This could be attributed to either a storage problem [33] or the amateur operator performing the cytology examination, as it requires experienced hands, good equipment, proper handling, and special staining techniques [34]. Owing to the nature of the primary disease in the selected population, patients usually presented with neutropenic fever and low platelet count, which were limiting factors to perform TBLB in all cases. TBLB was performed in 22 of 30 patients (73.3\%). The procedure was diagnostic in $100 \%$ of the 22 cases, of which (Fig. 3 ) seven cases (31.8\% of total biopsies, accounting for $23.3 \%$ of total cases) revealed progression of the primary disease, 12 cases $(54.5 \%$ of total biopsies, accounting for $40 \%$ of total cases) infections, and two cases (9\% of total biopsies; 6.6\% of total cases) showed complications of chemotherapeutic agents, and a pathological diagnosis of $P$. jiroveci pneumonia was reached in one case $(2.2 \%$ of total biopsies accounting for $3.3 \%$ of total cases). This is in contrast to Cazzadori et al. [9], where transbronchial biopsy provided conclusive diagnostic indications in only $55 \%$ of patients with hematologic malignancies.

TBLB when combined with BAL provides a high diagnostic yield especially in noninfectious etiologies of pulmonary infiltrates in patients with hematological malignancies [35].

In a retrospective study [36], only 11 of 95 immunocompromised patients with hematological disorders had sufficient platelet counts to allow TBLB, but the procedure was diagnostic in seven (64\%) of them. Similarly, a prospective study [37] conducted on 200 immunocompromised patients showed that TBLB was performed in only 11 patients but was diagnostic in six (55\%) of them, most of whom had their treatment changed as a result of the findings. In another prospective study [19], TBLB was possible in 45 of 104 immunocompromised patients. Overall, BAL had a diagnostic yield of $38 \%$. In the 45 patients who underwent TBLB, the diagnostic yield was $44 \%$ for TBLB and $70 \%$ for BAL plus TBLB. TBLB was the only investigation that provided the diagnosis in $28 \%$ of patients, of whom all but two had noninfectious infiltrates.

\section{Conclusion}

The combined diagnostic yield of BAL for microbiological examination and TBLB was higher than that of BAL for cytology examination. Thus, this study supports the role of FOB as the initial procedure of choice for identifying the nature of pulmonary infiltrates in patients with hematological malignancies. Because our results suggest that BAL and TBLB are complementary, we recommend performing TBLB whenever it is considered safe.

\section{Financial support and sponsorship \\ Nil.}

\section{Conflicts of interest}

There are no conflicts of interest.

\section{References}

1 Jones RJ, Armstrong SA. Cancer stem cells in hematopoietic malignancies. Biol Blood Marrow Transplant 2008; 14:12-16.

2 Nelson $\mathrm{MH}$, Paulos CM. Novel immunotherapies for hematologic malignancies. Immunol Rev 2015; 263:90-105.

3 Shorr A, Kollef $M$. The quick and the dead: the importance of rapid evaluation of infiltrates in the immunocompromised patient. Chest 2002; 122:9-12.

4 Azoulay É, Ayman OS. Respiratory infections in patients with hematological malignancies: pulmonary involvement in patients with haematological malignancies. Berlin Heidelberg: Springer-Verlag 2011; 10:9-38.

5 Bommart S, Bourdin A, Makinson A, Durand G, Micheau A, Monnin-Bares $V$, et al. Infectious chest complications in haematological malignancies. Diagn Interv Imaging 2013; 94:199-207.

6 Choi MH, Jung JI, Chung WD, Kim YJ, Lee SE, Han DH, et al. Acute pulmonary complications in patients with hematologic malignancies. Radiographics 2014; 34:1755-1768.

7 Shawgi S, Kumar L, Kochupillai V, Shukla NK, Broor S, Kapila K, et al. Evaluation of pulmonary infiltrates in patients with haematological malignancies using fibreoptic bronchoscopy and bronchoalveolar lavage. Indian J Med Paediatr Oncol 2004; 25:10-21.

8 Murro D, Slade JM, Gattuso P. Cytologic findings of hematologic malignancies in bronchoalveolar lavage fluid. J Am Soc Cytopathol 2015; 4:267-275.

9 Cazzadori A, Di Perri G, Todeschini G, Luzzati R, Boschiero L, Perona G, et al. Transbronchial biopsy in the diagnosis ofpulmonary infiltrates in immunocompromised patients. Chest 1995; 107:101-106.

10 Curley FJ, Johal JS, Burke ME, Fraire AE. Transbronchial lung biopsy: can specimen quality be predicted at the time of biopsy? Chest 1998; 113:1037-1041.

11 American Cancer Society. Cancer facts and figures 2014. Available at: http://www.cancer.org/acs/groups/content/@ research/documents/ document/acspc-041770.pdf. [Last accessed 2017 Oct 1]. 
12 The Most Frequent Cancers, All Aswan Governorate, Egypt, 2008 Available at: http://www.cancerregistry.gov.eg. [Last accessed 2017 Oct 1].

13 Ibrahim AS, Khaled HM, Mikhail NNH, Baraka H, Kamel H. Cancer incidence in Egypt: results of the national population-based cancer registry program. J Cancer Epidemiol 2014; 2014:437971.

14 Shirley MH, Sayeed S, Bames I, Finlayson A, Ali R. Incidence of hemtological malignancies by ethic group in England. $\mathrm{Br} J$ Hematol 2013; 163:465-477.

15 Ahmed S, Siddiqui AK. Pulmonary complications in chronic lymphocytic leukemia. Cancer 2003; 98:1912-1917.

16 Smith A, Crouch S, Lax S, Li J, Painter D, Howell D, et al. Lymphoma incidence, survival, and prevalence 2004-2014: sub-type analyses from the UK's: hematological malignancy research network. Br J Cancer 2015; 112:1575-1584.

17 Parikh SA, Rabe KG, Kay NE, Call TG, Ding W, Schwager S, et al. Chronic lymphocytic leukemia in young $<55$ years patient: a comprehensive analysis of prognostic factors and outcomes. Hematologica 2013; 99: 140-147.

18 Ward E, DeSantis C, Robbins A, Kohler B, Jemal A. Childhood and adolescent cancer statistics. CA Cancer J Clin 2014; 64:83-103.

19 Jain P, Sandur S, Meli Y, Arroliga AC, Stoller JK, Mehta AC. Role of flexible bronchoscopy in immunocompromised patients with lung infiltrates. Chest 2004; 125:712-722.

20 Tanaka N, Matsumoto T, Miura G, Emoto T, Matsunaga N. HRCT findings of chest complications in patients with leukemia. Eur Radiol 2002; 12:1512-1522.

21 Hofmeister CC, Czerlanis C, Forsythe S, Stiff PJ. Retrospective utility of bronchoscopy after hematopoietic stem cell transplant. Bone Marrow Transplant 2006; 38:693-698.

22 Poutsiaka DD, Price LL, Ucuzian A, Chan GW, Miller KB, Snydman DR. Blood stream infection after hematopoietic stem cell transplantation is associated with increased mortality. Bone Marrow Transplant 2007; 40 63-70.

23 Kotloff RM, Ahya VN, Crawford SW. Pulmonary complications of solid organ and hematopoietic stem cell transplantation. Am J Respir Crit Care Med 2004; 170:22-48.

24 Freifeld AG, Bow EJ, Sepkowitz KA, Boeckh MJ, Ito Jl, Mullen CA, et al. Clinical practice for the use of antimicrobial agents in neutropenic patients with cancer: update by the Infectious Diseases Society of America. Clin Infect Dis 2011; 52:e56-e93.
25 Tertemiz KC, OzgenAlpaydin A, Salman S, Alacacioglu I. Diagnostic yield of bronchoalveolar lavage in hematological malignancies. Eur Respir $J$ 2015; 46:2646.

26 Drent M, Wagenaar SS, Mulder PH, van Velzen-Blad H, Diamant M, van den Bosch JM. Bronchoalveolar lavage fluid profiles in sarcoidosis, tuberculosis and non-Hodgkin's and Hodgkin's disease. An evaluation of differences. Chest 1994; 105:514-519.

27 Zompi S, Couderc LJ, Cadranel J. Clonality analysis of alveolar B lymphocytes contributes to the diagnostic strategy in clinical suspicion of pulmonary lymphoma. Blood 2004; 103:3208-3218.

28 Poletti V, Romagna M, Gasponi A, Baruzzi G, Allen KA. Bronchoalveolar lavage in the diagnosis of low-grade, MALT type, B-cell lymphoma of the lung. Monaldi Arch Chest Dis 1995; 50:191-194.

29 Poletti V, Salvucci M, Zanchini R. The lung as a target organ in patients with hematologic disorders. Haematologica 2000; 85:855-864.

30 Semenzato G, Poletti V. Bronchoalveolar lavage in lung cancer Respiration 1992; 59:44-46.

31 Azoulay E, Fieux F, Moreau D, Thiery G, Rousselot P, Parrot A. Acute monocytic leukaemia presenting as acute respiratory failure. Am J Respir Crit Care Med 2003; 167:1329-1333.

32 Poletti V, Poletti G, Murer B. Bronchoalveolar lavage in malignancy. Semin Respir Crit Care Med 2007; 28:534-545.

33 Rennard SI, Ghafouri M, Thompson AB, Linder J, Vaughan W, Jones K, et al. Fractional processing of sequential bronchoalveolar lavage to separate bronchial and alveolar samples. Am Rev Respir Dis 1990; 141:208-217.

$34 \mathrm{Klech} \mathrm{H}$, Hutter $\mathrm{C}$. Clinical guidelines and indications for bronchoalveola lavage (BAL): report of the European Society of Pneumology Task Group on BAL. Eur Respir J 1990; 3:937-969.

35 Mulabecirovic A, Gaulhofer P, Auner HW, Popper H, Krause R, Hesse C, et al. Pulmonary infiltrates in patients with haematologic malignancies: transbronchial lung biopsy increases the diagnostic yield with respect to neoplastic infiltrates and toxic pneumonitis. Ann Hematol 2004; 83:420-422.

36 Hohenadel I, Kiworr M, Genitsariotis R, Zeidler D, Lorenz J. Role of bronchoalveolar lavage in immunocompromised patients with pneumonia treated with a broad spectrum antibiotic and antifungal regimen. Thorax 2001; 56:115-120.

37 Rano A, Agusti C, Jimenez P, Angrill J, Benito N, Daned C, et al. Pulmonary infiltrates in non-HIV immunocompromised patients: a diagnostic approach using non-invasive and bronchoscopic procedures. Thorax 2001; 56:379-387. 